\title{
NIVELES DE DEPRESIÓN Y ANSIEDAD EN ESTUDIANTES UNIVERSITARIOS DE LIMA METROPOLITANA
}

\author{
Marcelino Riveros Q. ${ }^{1}$, Héctor Hernández V. ${ }^{2}$, José Rivera B. ${ }^{3}$
}

(Recibido el 08/03/2007, aceptado el 11/03/2007)

\section{RESUMEN}

En esta investigación presentamos el nivel de ansiedad y depresión en los estudiantes universitarios de la UNMSM. La muestra representativa estuvo formada por 500 estudiantes universitarios de la UNMSM, distribuidos por género y por bloque académico, se evaluó la prevalencia de depresión y ansiedad mediante la prueba denominada HADS, constituida por siete ítemes para cada trastorno, con un puntaje de 0 a 3, traducida al español. Dado que dicha aprueba ha sido utilizada en América del Norte y países asiáticos, se partió del supuesto de que ya existe una tendencia que incluso es internacional. En Lima no existen trabajos con el HADS.

Palabras clave: Depresión, Ansiedad, HADS, Estudiante universitario, Prevalencia.

\begin{abstract}
In this investigation we display the level of anxiety and depression in university students of the UNMSM. The representative sample was formed by 500 university students of the UNMSM, distributed by sort and by academic block, in which we evaluated prevalence of depression and anxiety by means of denominated test HADS, constituted by seven items for each upheaval, with a pentane from 0 to 3, translated into Spanish. Since this test has been used in North America and Asian countries, it was assumed that there is already an international tendency. In Lima do not exist works with the HADS.
\end{abstract}

Keywords: Depression, Anxiety, HADS, University Student, Prevalence.

1 Docente Asociado, Facultad de Psicología de la UNMSM. E-mail: mriverosquiroz@yahoo.com

2 Docente Asociado, Facultad de Psicología de la UNMSM. E-mail: hhernandezv@unmsm.edu.pe

3 Docente Asociado, Facultad de Psicología de la UNMSM. E-mail: jriverab@unmsm.edu.pe 


\section{INTRODUCCIÓN}

La depresión es uno de los desórdenes clínicos más frecuentes en la población en general, actualmente existe una codependencia entre depresión y ansiedad; diversos marcos conceptuales que parten de la práctica clínica y de los hallazgos de las investigaciones intentan explicarla para intervenir sobre ella y de esta manera prevenir el suicidio.

En la actualidad la triada ansiedad, depresión y estrés está causando más muertes que el cáncer y el sida juntos.

La depresión se incluye en el grupo de los «trastornos afectivos», dado que una característica de la misma es la alteración de la capacidad afectiva, siendo no sólo la tristeza sino también la incapacidad de dar afecto o recibirlo de quienes nos rodean.

\section{Clasificación de los estados depresivos}

Los estados depresivos no son una entidad monolítica invariable. Si se aceptan las clasificaciones que se han hecho hasta el presente, se verá que han sido descritos más de cien tipos depresivos que se suponen distintos unos de otros. Esta abundancia puede confundir.

\section{Clasificación actual}

En esta se hace mayor hincapié en saber si el síndrome depresivo está o no relacionado con otras entidades y si cumple o no ciertos requisitos, como síntomas, signos, gravedad y tiempo de solución.

Depresiones secundarias: Son las relacionadas con otras enfermedades médicas o psiquiátricas. Es indispensable identificar el trastorno original que las provocó.

Secundarias en relación con otras alteraciones psiquiátricas.

- La esquizofrenia, tras la remisión del episodio agudo o en algunas manifestaciones de la esquizofrenia que se caracterizan por una actitud clínica negativa.

- Los estados de ansiedad aguda o generalizada se vuelve crónica con el tiempo y no se resuelve.

Los alcohólicos y otros adictos, al retirárseles el tóxico que les causa dependencia

Secundarias en relación con otras alteraciones médicas.

Depresiones primarias: Son las que no están ligadas a otro tipo de enfermedad médica o psiquiátrica. Aparecen de manera autónoma o una vez que se producen los desencadenamientos antes descritos. Las subdivisiones son de acuerdo con su sintomatología y su gravedad.

Depresiones primarias unipolares. Se presentan en fases. Cuando no se remedian, a los intervalos de normalidad siguen fases depresivas. Tanto la duración de la fase como el tiempo que transcurre entre una y otra varía de un individuo a otro. En algunas el episodio dura solo unas semanas; en otras se vuelve crónico con el paso de los años. Suelen aparecer a partir de los treinta años de edad, pero desaparecen con un tratamiento adecuado. 
Depresiones primarias bipolares. Las fases depresivas alternan con intervalos de normalidad, pero también con episodios maníacos. La manía es otra cara de la moneda de la depresión, los pacientes se muestran eufóricos, expresivos, son imperativos, se entregan a la verborrea, padecen insomnio, hacen gastos excesivos, se comprometen a hacer tareas irrealizables y se muestran irascibles cuando se les lleva la contraria. En casos graves pueden llegar a la agitación, a las ideas delirantes o a las alucinaciones.

\section{Identificación de los signos y síntomas de la depresión}

Estado de ánimo. Este se encuentra bajo, el enfermo puede darse cuenta de esto, aunque es frecuente que los familiares cercanos, lo hayan notado. El paciente es visto como más callado, distante, serio, aislado, o irritable. Esto último puede ser un dato significativo del cambio en el carácter del paciente. Estar poco tolerante y sentir que solo a él le ocurren todas las cosas malas o que es él quien las provoca. El estado de ánimo puede variar a lo largo del día. El paciente deprimido nota que hay una parte del día en que se siente más triste, por ejemplo la mañana, y conforme pasa el día, va sintiéndose mejor. Puede haber llanto, con frecuencia, puede presentarse al recordar experiencias negativas en la vida reciente o remota.

Pérdida del interés por situaciones o actividades que antes le producían placer. Esta es otra manifestación cardinal de la depresión. El paciente ya no se interesa por las actividades que antes le gustaba realizar. Por ejemplo: ir al cine, salir con amigos, oír música, leer, su propio trabajo. Esto puede deberse, a que ya no disfruta el efectuar esas actividades, o a que ya se le dificultan. Por ejemplo, si a una persona le gustaba leer, pero ahora le cuesta trabajo concentrarse y retener lo que está leyendo, y hace un gran esfuerzo en esto, entonces empieza a no ser una actividad agradable. Lo mismo es el asistir a una reunión o una fiesta, la dificultad para interactuar con los demás se ve magnificada, por el hecho, que ahora el enfermo se siente con poca capacidad para estar bien con sus amigos y familiares, por lo que se torna un suplicio, el ver a los demás reír y divertirse, cuando es algo que él o ella no pueden experimentar. El pedirle a los deprimidos que «le eche ganas»; «Que no se den por vencido»; «Que se esfuerce y socialice», es solicitarle que vaya a contractarse y a sentirse peor.

Sentimientos de culpa. Este tipo de síntoma es muy frecuente en el deprimido. Ellos pueden pensar que están deprimidos por cosas o situaciones que hicieron o dejaron de hacer en el pasado. Aun más pueden llegar a sentir que el estar deprimido es una forma de castigo, y que están expiando sus culpas a través de su enfermedad. Finalmente en algunas formas de depresión psicótica, el enfermo puede tener ideas delirantes (ideas fuera del juicio de realidad), de que están pagando no solo con sus culpas, sino las culpas de alguien más o que están expiando los pecados de tal o cual grupo de seres marginados, etc. Pueden incluso existir alucinaciones auditivas, que los acusen e insulten.

Ideación suicida. Los enfermos con depresión se suicidan con una alta frecuencia. El médico puede tener miedo de preguntar respecto a esto, porque puede suponer que el enfermo no ha pensado en eso, o por lo menos no ha pensado en eso formalmente, y que al hacer semiología en esta área, puede «despertar» la ideación suicida. Pero el enfermo ya lo ha pensado y es más, él desea que se le interrogue al respecto. Porque, desea y necesita que lo ayuden a no llevar a cabo este tipo de ideas. 
Insomnio y otros trastornos del sueño en la depresión. El insomnio es la manifestación de sueño insuficiente o poco reparador. En el caso de los enfermos deprimidos, la forma más típica, es el insomnio de la última parte de la noche, también llamado insomnio terminal o tardío. El paciente se despierta a las 03:00 de la madrugada, por ejemplo, y no puede volverse a dormir. En esas horas de soledad nocturna, el paciente inicia con una serie de pensamientos pesimistas y de impotencia, que le impiden dormir nuevamente («un día más»; «ni siquiera esto puedo hacer bien»; «no voy a poder continuar con esto»). Despertarse una hora más temprano, del horario habitual, que solía tener el paciente, antes del inicio de su depresión, es considerado como despertar matutino prematuro o insomnio terminal. En este sentido, una pregunta clave es: $i$ a que hora solía despertarse por últimas vez en la mañana, para levantarse, antes de estar deprimido? Y, ¿a qué hora en promedio se ha estado despertando sin poder dormir nuevamente, en la última semana?

Algunos pacientes con depresión pueden tener datos de insomnio inicial (incapacidad para iniciar el sueño por más de 30 minutos, en el horario acostumbrado) o insomnio terminal (fragmentación del sueño, con despertares de más de 20 minutos o levantarse de la cama, sin tener la necesidad de ir al baño). En un laboratorio de sueño, el enfermo con depresión tiene una serie de alteraciones, de las fases o estadios de sueño, como son: menor cantidad de fases de sueño III y IV (sueño delta), un inicio rápido al sueño de movimientos oculares rápidos (latencia acortada al SMOR); fragmentación del sueño y despertar matutino prematuro.

Sin embargo, puede haber algunos enfermos que, en vez de presentar una baja en calidad y cantidad de sueño, tienen lo opuesto, es decir un exceso de sueño. Estas son personas que duermen de nueve a diez horas, y que refieren que entre más duermen, más deprimidos se sienten, con gran estado de adinamia, debilidad y lentitud psicomotriz.

Disminución de energía. El paciente refiere que tiene gran dificultad para moverse, para arreglarse, asearse, para iniciar labores mínimas, o que requiere de un gran esfuerzo para llevarlas a cabo: «Siento como si estuviera con las pilas bajas»; «No me dan ganas de nada, solo quiero estar en la cama tumbado sin hacer nada»; «Las cosas las hago como en cámara lenta». Aquí hay que evaluar qué cosas ya no puede hacer el paciente, y esto va desde que requiere un gran esfuerzo para trabajar, para su aliño, hasta dejar de trabajar y estar únicamente acostado o en una posición fija todo el tiempo. Los enfermos pueden llegar a un tipo de estupor, que dificulta mucho la entrevista clínica, con una gran lentitud para responder, o solo lo hacen con movimientos de cabeza. El mutismo o estupor del deprimido se diferencia de la catatonia, que también es un tipo de inmovilidad, en que en esta última el enfermo tiene un signo llamado: «Flexibilidad cérea» (de cera de las velas), en donde se le coloca en posiciones antigravitatorias incómodas, por ejemplo con un brazo levantado, como de estatua de cera, y permanece en ese estado por un largo rato. Situación que no ocurre con el enfermo deprimido.

Agitación psicomotriz y ansiedad psíquica. Estas son dos manifestaciones que se pueden presentar en depresiones, en donde hay un componente sintomático ansioso importante. El médico puede evaluar desde la inspección general al paciente, su estado de agitación psicomotriz, de estar presente, el paciente mueve las manos o los pies de 
manera inquieta, juguetea con los cabellos, se seca las manos, se levanta y sienta, etc. Además el paciente puede referir que se siente «nervioso», agitado, que no puede estar tranquilo, que se está preocupando mucho por cosas pequeñas que antes no le preocupaban, y aquí habrá que pedirle ejemplos de las mismas: «Ahora me preocupo mucho por la hora que llega mi marido, si no me habla dos o tres veces al día estoy nerviosa, esto no me sucedía antes».

Malestares físicos. Es común que el paciente con depresión presente una serie de malestares, poco sistematizados, pero que pueden ser severos: cefalea, dispepsias, boca seca, náusea, suspiros excesivos, sensación de respiraciones que no son suficientes, malestares musculares, etc.

Alteraciones gastrointestinales y pérdida de peso. Es frecuente que los pacientes con depresión presenten baja en su apetito, con una disminución significativa de su peso corporal. Esto se puede cuantificar en kilos, si es que el paciente se ha pesado o en tallas de ropa, ya que el paciente, siente que su ropa le queda holgada. Una pérdida de peso de más de $5 \mathrm{~kg}$, sin dieta, deberá de ser estudiada, descartando problemas médicos, trastornos de la alimentación, sobre todo, si la paciente es mujer (v.g. anorexia o bulimia) y dietas. Por otro lado, algunos pacientes con depresión pueden aumentar de peso, esto se ve más frecuentemente en los enfermos con sueño excesivo, apetencia por alimentos ricos en carbohidratos e inhibición psicomotriz. A este cuadro que se ha descrito se le denomina: depresión afectiva estacional, porque es común que con esas características clínicas, se presente con más frecuencia en el invierno. También se le ha llamado depresión atípica.

Disminución del deseo sexual. Se ha notado una baja en el deseo sexual (libido), o disfunción eréctil en el hombre y anorgasmia en la mujer.

Manifestaciones diversas. Estas pueden ser de tipo hipocondríaco, estar buscando explicaciones en el funcionamiento de sus diferentes órganos y sistemas, acudir más frecuentemente al médico con dolencias poco sistematizadas. También pueden haber datos obsesivo-compulsivos, es decir, el paciente tiene pensamientos repetitivos que no puede apartar de su cabeza, y que son reconocidos como absurdos y la repetición de actos motores o rituales, varias veces, para estar seguro de que se hacían y estaban bien hechos. En el primer caso son ideas parásitas que no se van de la cabeza y que pueden generar ansiedad a los pacientes. En el segundo, el paciente tiene que estar revisando cosas y situaciones, para estar seguro de que las puertas y ventanas estén bien cerradas, o repetir operaciones, como el aseado, varias veces para estar seguro que estén hechos.

\section{Trastorno de ansiedad}

La ansiedad es un mecanismo adaptativo natural que nos permite ponernos alerta ante sucesos comprometidos. En realidad, un cierto grado de ansiedad proporciona un componente adecuado de precaución en situaciones especialmente peligrosas. Una ansiedad moderada puede ayudarnos a mantenemos concentrados y afrontar los retos que tenemos por delante.

En ocasiones, sin embargo, el sistema de respuesta a la ansiedad se ve desbordado y funciona incorrectamente. Más concretamente, la ansiedad es desproporcionada con la 
situación e incluso, a veces, se presenta en ausencia de cualquier peligro ostensible. El sujeto se siente paralizado con un sentimiento de indefensión y, en general, se produce un deterioro del funcionamiento psicosocial y fisiológico. Se dice que cuando la ansiedad se presenta en momentos inadecuados o es tan intensa y duradera que interfiere con las actividades normales de la persona, entonces se le considera como un trastorno.

\section{Problemas de salud mental en universitarios}

En el año 2003, en el estudio: Características sociodemográficas y niveles de depresión en estudiantes ingresantes a la UNMSM a través de la escala CES-D, realizado por Marcelino Riveros Quiroz, se encontró que de una muestra 382 alumnos el $40 \%$ está propenso a desarrollar un cuadro depresivo, de los cuales 37 casos obtuvieron puntajes elevados de depresión y 118 casos con puntajes intermedios o propensos a desarrollar cuadros depresivos.

En el año 2003, Amézquita Medina, María; González Pérez, Rosa; Zuluaga Mejía, Diego evaluaron una muestra de 625 estudiantes de los diferentes programas académicos seleccionados al azar, con los inventarios de ansiedad y depresión de Beck y una encuesta semiestructurada. Fue una investigación cuantitativa y transversal con un diseño descriptivo. Las variables dependientes fueron depresión, ansiedad y comportamiento suicida. Las variables independientes fueron: edad, sexo, estado civil, facultad, programa, nivel académico y socioeconómico, actividad laboral, procedencia, residencia actual, pérdidas y satisfacción con la carrera.

Además se estableció una asociación entre estos dos conjuntos de variables. Para el análisis e interpretación de los datos se utilizó la estadística descriptiva e inferencial (chi cuadrada, tabla de contingencia $2 \times 2$ ). Se encontró una prevalencia del 49,8\% para depresión. 58,1\% para ansiedad y $41 \%$ para ideación suicida. Entre las asociaciones para depresión y ansiedad que resultaron estadísticamente significativas fueron: Programa de Desarrollo Familiar: sexo femenino, pérdidas afectivas; Académicas, económicas y de salud: insatisfacción con la carrera; dificultades en las relaciones con compañeros y docentes, responsabilidades académicas, y comportamiento suicida.

También, según Manelic RH y Ortega-Soto HA (1995), en los estudiantes universitarios la depresión puede constituir un problema importante, pues al malestar psicológico del individuo se agrega la imposibilidad de obtener un rendimiento académico satisfactorio ya que, como se sabe, las funciones cognoscitivas se alteran sensiblemente cuando se presenta esta entidad nosológica.

Varios estudios indican que la frecuencia de los cuadros depresivos es mayor en la población estudiantil que en la general, y que en ese primer grupo predominan las mujeres y los estudiantes de licenciaturas «difíciles» (vg ingeniería, medicina, etc.).

En este estudio se utilizó el Inventario de Depresión de Beck (11313) para detectar los casos que tenían algún síndrome depresivo, en una muestra aleatoria, proporcional por sexo y carrera, del Plantel Aragón de la Escuela Nacional de Estudios Profesionales de la Universidad Nacional Autónoma de México. Del total de 1026 sujetos evaluados (el 10\% de la población total), 378 eran mujeres y el resto hombres.

Utilizando el punto de corte $>14$ puntos para considerar la presencia de un síndrome depresivo, se encontró una prevalencia general de $11.8 \%, 69$ hombres y 51 mujeres: la 
diferencia no fue significativa. Pero no se encontraron diferencias en la prevalencia entre los sujetos de las diferentes carreras que se imparten en el plantel. Son resultados que concuerdan con los de otros estudios, en el que la frecuencia de los cuadros depresivos en la población universitaria es alta, por lo que es recomendable implementar servicios de atención psicológica en los centros de estudios superiores.

Entre los factores que generan depresión entre universitarios se encuentra al elevado costo de los estudios y un mercado laboral muy limitado, es decir, primero un gran esfuerzo y luego la frustración; como se puede concluir de la información proporcionada por la agencia de prensa Elmundo.es, según la cual un $25 \%$ de los estudiantes universitarios de Beijing sufre depresión y muchos tratan de suicidarse, constituyendo este un incremento relativamente reciente debido a la presión financiera, la competición académica feroz y un ajustado mercado de trabajo para graduados.

Al menos 100000 estudiantes universitarios sufren depresión mayor y 19000 han cometido suicidio en 2004, según el departamento oficial de salud. Asimismo, «El desconocimiento sobre la enfermedad resulta en una falta de comprensión y antipatía hacia las personas que padecen depresión», señala el departamento.

El precio de los estudios superiores en China, que eran gratuitos hasta hace menos de 20 años, se ha disparado. Cuatro años de estudios universitarios cuestan como mínimo 28000 yuanes (3500 dólares), que equivale aproximadamente a los ingresos de un granjero durante 35 años, según informa la agencia de noticias Xinhua.

Pero este no es el único problema, ya que además del gran esfuerzo que supone capear estas duras condiciones económicas, muchos estudiantes tienen que enfrentarse a un mercado laboral incapaz de absorberlos una vez graduados. Ante esta situación, el gobierno chino ha declarado que tiene en cuenta la situación y actuará poniendo límites a las admisiones a la educación superior, así como recortando los precios de los comedores universitarios.

En México, María Guadalupe Rosete Mohedano (2003) investigó la relación entre salud mental y rendimiento académico en estudiantes universitarios, en las carreras de medicina, psicología y odontología, bajo la premisa de que «Los problemas de salud mental en las últimas décadas representan un reto para la Salud Pública». Sin precisar cifras, es por todos conocidos el incremento en las tasas de depresión, suicidios, hogares desintegrados, violencia intrafamiliar, drogadicción en sus diferentes modalidades e incluso de psicosis. Es sabido que toda persona, evidentemente, reacciona ante cualquier cambio importante de la vida; pero cuando estas reacciones alcanzan determinado grado de impacto y no son resueltas por los canales adecuados, propios de una personalidad madura, pueden considerarse como trastornos emocionales y, por consiguiente, de salud mental [1]. Existen grupos de personas que son más vulnerables, entre ellos se encuentran los adolescentes, en quienes aparecen otras formas de conducta y se expresan como problemas de relaciones familiares, sexuales, escolares, de trabajo; muchas veces se canalizan hacia hábitos inadecuados como las adicciones, y en los adolescentes estudiantes el problema principalmente se localiza en el rendimiento escolar. Cita que «Es importante resaltar que de 576 alumnos reportados como positivos, el 32,6\% correspondieron a la carrera de Enfermería, el segundo lugar lo ocupó la carrera de Psicología, con un $21,8 \%$; el tercer lugar, fue ocupado por la carrera de Odontología, con un $12 \%$, seguido de Medicina, $9,4 \%$ y QFB, $9,0 \%$, ocupando los últimos sitios: Ingeniería Química, 6\% y Biología, 2,9\%. Estas tres últimas carreras tuvieron los índices más altos de reprobación y de abandono de la carrera. 
Llama la atención que sólo el 16\% de los alumnos que resultaron positivos en la prueba de Goldberg durante los años reportados, hayan concluido la carrera, por lo que cinco de cada seis alumnos eran irregulares y cerca del 35\% de esta población había abandonado la carrera.

En su propia investigación en la Fes Zaragoza, con estudios de Medicina, Odontología y Psicología, encontró como un dato de sumo interés que existen otros datos cualitativos que son más reveladores, a más del $60 \%$ de los alumnos positivos en Goldgerg de las generaciones estudiadas se les aplicó EPM, donde se encontró en su psicodinamia un alto porcentaje de dificultades emocionales, sobre todo, depresivas y de angustia y con un alto índice de ideas suicidas, con elevados índices de bajo desempeño intelectual, atribuibles a las perturbaciones emocionales y a una deficiente maduración de la personalidad, que implica poca capacidad para las tareas por deficiencia en la integración psicomotriz, así como en la correlación del pensamiento abstracto con hechos reales, pobre autoobservación y fundamentación del proceso de identidad, no solamente sexual sino también como sujetos sociales, existiendo en muchos casos factores que limitaron el desempeño al estar ocupados en atender su inestabilidad emocional y lo que menos les preocupaba es estudiar. El miedo a la soledad y al ostracismo en muchos de ellos no les facilitaba una integración a su comunidad estudiantil, no sólo con su carrera, sino también con las otras carreras.

Se detectó, además, en los alumnos que acudieron al servicio una frecuencia alta de pertenecer a hogares desintegrados, de alcoholismo en alguno o ambos padres, baja autoestima, poca o nula estimulación para actividades socioculturales, deficientes hábitos de estudio, escasos recursos económicos para satisfacer las necesidades básicas, de educación y aún menos de recreación y esparcimiento. El ambiente de estos estudiantes se hace propicio para adherirse a algún tipo de droga, siendo las más frecuentes el alcohol y el tabaco, sin dejar de estar ausente la marihuana, los solventes, algún fármaco y la cocaína.

Los factores psicosociales son determinantes para el amor a la vida, la condición de abundancia contra la insuficiencia, tanto económica como cultural o emocional; facilitan o no el desarrollo que permite trascender la vida. En la medida en que el hombre gasta su energía luchando contra la escasez, para obtener los recursos mínimos necesarios para sobrevivir, insuficiente tiempo le quedará para realizar actividades de recreación y esparcimiento, no tendrá energía para satisfacer sus necesidades emocionales, provocando resentimientos, malestar, inconformidad, hostilidad a la vida. Cuando se crece en sistemas enajenantes, limitado en educación, con poca estimulación sociocultural, sin conocer otras formas de vida, se transitará con una pobreza espiritual, sin fe y esperanza, sin libertad para crear, construir, admirar y aventurarse, se negará el amor que es la preocupación activa por la vida y el crecimiento de lo que amamos, con sus elementos básicos como son el cuidado, responsabilidad, respeto y conocimiento (Fromm 1956).

Como datos cuantitativos encuentra que en medicina: Los alumnos de medicina clasificados como positivos presentaron el mayor número de módulos no acreditados, el $23 \%$ no había aprobado uno solo de los módulos; es decir, para las fechas en que se hizo el estudio estos sujetos habían ya abandonado la carrera, el 13\% de ellos tenían menos del 25\% de los módulos cubiertos y solamente el 4,3\% habían acreditado completo el plan de estudios. El porcentaje de alumnos que abandonaron la carrera fue de $37 \%$.

La calificación con la que egresaron la mayoría de los estudiantes fue de 7,3, que sugiere la imposibilidad del ingreso a una especialidad. 
Los alumnos de medicina son los que presentan el mayor número de exámenes extraordinarios, que oscila de cinco a diez exámenes por modulo.

Psicología: El 26\% de los alumnos había acreditado más del 75\% de los módulos y un 17\% ya había acreditado el total de ellos, son los que con mayor frecuencia repiten cursos; existieron alumnos que hasta en diez módulos se habían inscrito dos veces, lo que prolonga hasta catorce semestres la conclusión de sus estudios.

Se observó que los alumnos de la carrera de Psicología acudían con mayor frecuencia al SSMyP y presentaban diferentes tipos de adicciones y problemáticas centradas en disfunciones familiares, se observó que lograban percibir su situación fantaseando que a través de la carrera resolverían su neurosis y los enigmas familiares.

Los egresados de esta carrera fueron los que obtuvieron el mayor promedio final: 7,5; sin embargo, es insuficiente para ingresar a estudios de postgrado.

Odontología: Los alumnos de esta carrera desertan en mayor proporción (42\%) con relación a las otras dos carreras; así también en un porcentaje mayor (28\%) habían acreditado el $100 \%$ del plan de estudios. Se observó también un porcentaje alto (11\%) de alumnos con cero acreditación, es decir, se inscribieron al primer semestre, pero no lo concluyeron.

Los alumnos de odontología abandonan más fácilmente la carrera, sobre todo en los primeros semestres, lo que permitió que los alumnos que se quedaron tuvieran el menor porcentaje de exámenes extraordinarios presentados. El promedio de calificación final fue de 7,1.

\section{MÉTODO}

\section{Diseño de Investigación}

La presente es una investigación descriptiva, ex post facto.

\section{Muestra}

Se utilizó una muestra representativa formada por 500 estudiantes universitarios de la UNMSM, distribuidos por género y por bloque académico.

Según el último censo de estudiantes de pregrado, efectuado por la Oficina Técnica del Estudiante, la población estudiantil se distribuye de la siguiente manera:

Cuadro 1. Alumnos de pregrado de la UNMSM según sexo.

\begin{tabular}{lcc}
\hline & Total & Porcentaje \\
\hline Masculino & 15684 & \\
Femenino & 12254 & \\
Alumnos censados & 27938 & \\
\hline
\end{tabular}

Fuente: Oficina Técnica del Estudiante-UNSMM, Censo de alumnos de pregrado. 
Cuadro 2. Alumnos de pregrado de la UNMSM distribuidos por sexo, según bloque académico.

\begin{tabular}{lccccccc}
\hline & \multicolumn{4}{c}{ Sexo } & \multicolumn{2}{c}{ Total } \\
\cline { 2 - 5 } & \multicolumn{2}{c}{ Masculino } & \multicolumn{2}{c}{ Femenino } & & \\
\cline { 2 - 5 } Ciencias de la salud & Total & Porc. & Total & Porc. & & Total & Porc. \\
\cline { 2 - 6 } Humanidades & 2767 & $46,2 \%$ & 3216 & $53,8 \%$ & 5983 & $100,0 \%$ \\
Ciencias básicas & 4166 & $48,9 \%$ & 4356 & $51,1 \%$ & & 8522 & $100,0 \%$ \\
Ciencias empresariales & 2759 & $52,7 \%$ & 2481 & $47,3 \%$ & 5240 & $100,0 \%$ \\
Ingenierías & 4579 & $77,9 \%$ & 1298 & $22,1 \%$ & 5877 & $100,0 \%$ \\
Total & 15684 & $56,1 \%$ & 12254 & $43,9 \%$ & 27938 & $100,0 \%$ \\
\hline
\end{tabular}

Fuente: Oficina Técnica del Estudiante-UNSMM, Censo de alumnos de pregrado.

El diseño muestral es de tipo aleatorio con un nivel de confianza del 0,05 y por tanto quedó establecido de la siguiente manera:

Cuadro 3. Muestra de estudiantes de la UNMSM en función del sexo, según bloque académico.

\begin{tabular}{|c|c|c|c|c|c|c|}
\hline & \multicolumn{4}{|c|}{ Sexo } & \multicolumn{2}{|c|}{ Total } \\
\hline & \multicolumn{2}{|c|}{ Masculino } & \multicolumn{2}{|c|}{ Femenino } & \multirow[b]{2}{*}{ Total } & \multirow[b]{2}{*}{ Porc. } \\
\hline & Total & Porc. & Total & Porc. & & \\
\hline Ciencias de la salud & 49 & 46,20 & 58 & 53,80 & 107 & 21,42 \\
\hline Humanidades & 75 & 48,90 & 78 & 51,10 & 153 & 30,50 \\
\hline Ciencias básicas & 25 & 61,00 & 16 & 39,00 & 41 & 8,29 \\
\hline Ciencias empresariales & 49 & 52,70 & 44 & 47,30 & 94 & 18,76 \\
\hline Ingenierías & 82 & 77,90 & 23 & 22,10 & 105 & 21,04 \\
\hline Total & 281 & 56,20 & 219 & 43,80 & 500 & 100,00 \\
\hline
\end{tabular}

\section{Instrumento}

El instrumento utilizado es la escala HADS formada por 14 ítemes, 7 para cada trastorno, con una escala de respuestas de 0 a 3 . Dicha escala ha sido probada ya en diferentes países y es de alta difusión.

En Gran Bretaña, Crawford JR, Henry JD, Crombie C, Taylor EP se propusieron obtener los datos normativos de la Escala de Ansiedad y De presión Hospitalaria (HADS). Mediante un diseño de medidas repetidas y correlacionales, el HADS fue administrado a una población no clínica ampliamente representativa de la población general adulta de Gran Bretaña (N $=1.792$ ) en términos de su distribución por edad, género y ocupación. Se usó el análisis correlacional para determinar la influencia de variables demográficas en los resultados del HADS. Se encontró que las variables demográficas mostraban una modesta influencia en las puntuaciones del HADS.

La fiabilidad del HADS es aceptable; las escalas de ansiedad y depresión correlacionan de forma moderada (.53). Concluyeron que los datos normativos presentados permiten a los 
clínicos valorar cualquier puntuación del HADS y proporcionan una información suplementaria a los puntos de corte existentes.

\section{RESULTADOS}

Los resultados son una manifestación más de las tendencias que envuelven a la depresión y a la ansiedad, en ese sentido, la población estudiantil de la UNMSM, aparece como representativa de la población del país. Si lo observado anteriormente debía considerarse como algo grave, lo que se presenta en este trabajo justifica dicha preocupación. En realidad no se esperaban mejores resultados y es por tanto una señal de que la prevención es indispensable y se precisa de refinar los procedimientos, considerando las variables ambientales y las oportunidades laborales, así como la aplicación de normas de baja ambigüedad.

Cuadro 4. Depresión en una muestra de estudiantes de ambos sexos de la UNMSM.

\begin{tabular}{lccccc}
\hline & Normal & $\begin{array}{c}\text { Depresión } \\
\text { leve }\end{array}$ & $\begin{array}{c}\text { Depresión } \\
\text { Moderada }\end{array}$ & $\begin{array}{c}\text { Depresión } \\
\text { severa }\end{array}$ & Total \\
\hline \multirow{2}{*}{ Femenino } & 176 & 34 & 8 & 2 & 219 \\
& $80,50 \%$ & $15,35 \%$ & $3,45 \%$ & $0,70 \%$ & $100 \%$ \\
\hline \multirow{2}{*}{ Masculino } & 258 & 15 & 6 & 1 & 281 \\
& $91,95 \%$ & $5,30 \%$ & $2,30 \%$ & $0,50 \%$ & $100 \%$ \\
\hline \multirow{2}{*}{ Total } & 435 & 49 & 14 & 3 & 500 \\
& $86,93 \%$ & $9,70 \%$ & $2,80 \%$ & $0,05 \%$ & $100,00 \%$ \\
\hline
\end{tabular}

Cuadro 5. Valores promedios de la ansiedad en una muestra de estudiantes de ambos sexos de la UNMSM.

\begin{tabular}{lcccc}
\hline $\begin{array}{c}\text { Ansiedad } \\
\text { social }\end{array}$ & N & $\begin{array}{c}\text { Medidas } \\
\text { mínimo }\end{array}$ & $\begin{array}{c}\text { Puntaje } \\
\text { mínimo }\end{array}$ & $\begin{array}{c}\text { Puntaje } \\
\text { máximo }\end{array}$ \\
\hline Femenino & 219 & 9.25 & 1 & 18 \\
Masculino & 281 & 8.97 & 0 & 21 \\
Total & 500 & 9.11 & 0 & 21 \\
\hline
\end{tabular}

\section{DISCUSIÓN}

Los resultados encontrados son coherentes con los estudios efectuados en países en los que los estudios universitarios son el medio para alcanzar la realización personal, pero ya desde su ingreso están presentes factores que contribuirán a hacerlos más vulnerables frente a los diferentes escenarios que le plantean los estudios superiores; entre los antecedentes están los aspectos familiares y psicosociales, con las patología que existen en su hogar y en con respecto a los estudios mismos, su costo, la exigencia y las oportunidades laborales. Con respecto a la UNMSM es poco lo que se puede decir en la actualidad, si se tiene en cuenta que se requieren de investigaciones a las que los estudiantes respondan sin hacer especulaciones sobre la utilidad que las autoridades van a hacer de ellas, en un escenario en el que se puede afirmar que no están prestando mayor atención por las condiciones en las que egresarán, desde el punto de vista formativo profesional. 


\section{CONCLUSIONES}

- La depresión se manifiesta con intensidad mayor en mujeres que en varones: $15,35 \%$ (depresión leve) y 2,30\% (depresión moderada), frente a un 5,30 \% (depresión leve) y un 2,30\% (depresión moderada) de los varones.

- La ansiedad es también mayor en mujeres que en hombres, con una media de 9,25 frente a 8,97 .

\section{RECOMENDACIONES}

Si existe un real interés en el éxito del estudiante en su carrera profesional, lo cual incluye su ingreso al campo laboral realizar nuevas investigaciones al respecto, incidiendo en el estado de salud mental, para exigir un rendimiento académico que eleve las probabilidades de inserción laboral, con contenidos curriculares acordes con el mercado de trabajo.

Crear condiciones académicas, en todos los aspectos pertinentes que permitan lograr el objetivo previo.

\section{REFERENCIAS BIBLIOGRÁFICAS}

1. Amézquita, M.; González, R.; Zuluaga, D. (2003). Prevalencia de la depresión en los estudiantes universitarios: una primera aproximación y algunas aportaciones metodológicas.

2. Aylard, P.; Gooding, J.; Nickura, P. (1987). Avalidation of three Anxiety and Depresión Self-assessment Scales. Journal of Psicosomatic Research; 31:261-268.

3. Crawford, J.; Henry, D.; Crombie, C.; Taylor, E. (2001). Psychol; 40(Pt 4):429-34. (Ref. 02-01).

4. Goldberg, D.; Williams, P. (1988). The Users Guide to the General Hearth Questionnaire. NFER/Nelson. Slouh.

5. Manelic, R.; Ortega, H. (1995). La depresión en los estudiantes universitarios de la Escuela Nacional de Estudios Profesionales Plantel Aragón, en Salud Ment; 18(2); 31-34.

6. Riveros, M. (2003). Características sociodemográficas y niveles de depresión en estudiantes ingresantes a la UNMSM a través de la escala CES - D.

7. Snaith, R. (1992). Availability of the Hospital Anxiety Depression Scale. Br J Psychiatry, 161-:422.

8. Vallejo, J.; Gastón, C. (1991). Trastornos afectivos: Ansiedad y depresión. Barcelona: Edit. Salvat.

9. Zigmond, A. ( 1983). Snaith, R. The Hospital Anxiety and Depresion Scale. Acta Psychiart Scand, 67: 361-370. 